\title{
Optimal feedback control in first-passage resetting
}

\author{
D. Lunz \\ E-mail: davin.lunz@polytechnique.edu \\ INRIA Saclay - Île de France, 91120 Palaiseau, France \\ École Polytechnique, CMAP 91128 Palaiseau, France \\ Institut Pasteur, 75015 Paris, France
}

\begin{abstract}
We study a diffusion process on a finite interval under the influence of a controllable drift where the particle resets to the left-hand side upon reaching the right-hand side. Assigning a pay-off for being nearer the right-hand side, but a penalty for reaching it, induces an inherent trade-off. We seek the drift feedback that maximises the long-term reward. By reducing the problem to a constrained variational problem we deduce that, for a wide class of problems, the optimal feedback law is remarkably straightforward: below a threshold state exert maximum drift; beyond the threshold exert minimum drift.
\end{abstract}

\section{Introduction}

Diffusion processes, describing the evolution of a quantity under the influence of random fluctuations, are ubiquitous across the physical, biological, social, and computational sciences $[1,2]$. Many such processes undergo drastic changes in state that are more rapid than the associated stochastic fluctuations but are well described by jumps that "reset" the process. For example, gene expression requires a chemical reaction to occur at a specific binding location along a DNA strand. A reactant in "search" of this location undertakes a random walk along the DNA strand driven by molecular diffusion, combined with discontinuous jumps due to association-dissociation events [3, 4, 5]. Ecological populations are well described by stochastic birth-death processes alongside sharp intermittent influxes or outfluxes due to immigration or catastrophes $[6,7,8,9]$. A queue of jobs awaiting allocation accumulates under stochastic arrival dynamics, such as in a telecommunications, manufacturing, or computing context. Jumps may occur due to catastrophic failure and subsequent system reset $[10,11,12]$. Sporadically resetting randomised algorithms found early application in reducing runtime $[13,14,15]$. More recently, the same idea has been adapted to searching physical space, as an animal might do in search of food or shelter [16]. Resetting the random walk guiding such a search by jumping back to an initial location can reduce first-passage times to a target $[17,18,19]$ (the firstpassage time to a target is the earliest time the state of the stochastic process reaches a prescribed region of state space). Enumerate extensions have been investigated, including higher dimensional space [20], adaptive searching [21], and restart with branching [22]. Many of these processes exhibit universal characteristics [23, 24] and can give rise to stationary power-law probability distributions underpinning a host of physical and social phenomena [25]. Refs. [26, 27, 28] provide more extensive references and historical context. 
The common theme threading this constellation of processes is a system evolving stochastically whilst undergoing intermittent reset events. In the cases of algorithms and search (including the gene expression mechanism described above), the focus has been to determine the timing structure of resets (the reset rate, or more complex timedependent structure) that minimises the expected time to an outcome [24, 29, 30, 31]. In population control, the reset timing that minimises the expected cost of a pest invasion has been studied [32]. In contrast to time-dependent resets, diffusion processes may have state-dependent resets, for example, resetting upon reaching the boundary of a domain [33, 34, 35, 36].

One particularly useful model introduced in Ref. [37] considers a particle $x$ diffusing in a one-dimensional bounded interval $x \in[0, L]$ that is reset to $x=0$ when the system reaches the critical state $x=L$. A pay-off is associated with being closer to the critical state while a cost is incurred for each reset. The process is controlled by a constant drift $v$, which leads to the optimisation problem: what drift minimises the long-term cost? This model can capture a power grid or production process, for example, where the system state $x$ represents the production rate and system failure occurs beyond the critical state (the system overheats, a machine jams/breaks, etc.). The optimality structure arises since, on the one hand, it is advantageous to be operating at high production rate, while on the other hand, operating near the system failure rate leads to costly reset events that cause the system to jump to low production rate.

A power station or factory process is more realistically going to accelerate production upon reset, but not near system failure. In this letter, our aim is to incorporate such strategies in a feedback-control drift $v(x)$. In this setting, optimising the long-term reward becomes an infinite-dimensional problem. While it may be difficult for some production processes to continuously update the control as the system state changes, remarkably, the infinite-dimensional problem admits an eminently implementable solution: maximise production rates until some threshold rate $x=x_{0}$, beyond which minimise any further production rate increase. Thus the problem reduces to a one-dimensional optimisation problem: find the threshold $x_{0}$ that maximises the long-term reward.

\section{Feedback control formulation}

Consider a particle $x$ moving on a finite interval $x \in[0, L]$ under a constant diffusion of magnitude $D$ and a state-dependent drift $v(x)$. Upon reaching the right-hand side $x=L$, the particle resets to the origin $x=0$, which acts as a reflecting boundary. The probability density $P$ of the system state $x$ is governed by

$$
\frac{\partial P}{\partial t}+\frac{\partial \Phi}{\partial x}=\delta(x)\left(\left.\Phi\right|_{x=L}\right), \quad 0<x<L,
$$

subject to boundary and initial conditions

$$
\left(\left.\Phi\right|_{x=0}\right)-\delta(t)=P(L, t)=P(x, 0)=0,
$$

where $\Phi$ denotes the flux

$$
\Phi=v(x) P-D \frac{\partial P}{\partial x} .
$$


We associate a reward with the system state, accumulated at a rate proportional to $x$, and a fixed cost $C$ to each reset event, to give the long-term reward function

$$
\mathcal{R}=\lim _{T \rightarrow \infty} \frac{1}{T}\left[\frac{1}{L} \int_{0}^{T} x(t) \mathrm{d} t-C N(T)\right],
$$

where $N(T)$ is the number of resets that occurred up to time $t=T$.

No realistic production process can be accelerated or decelerated infinitely fast and thus the drift must be bounded: $u \leq v(x) \leq U$. Our goal is to maximise $\mathcal{R}$ over the set of functions bounded within $[u, U]$.

We adopt the renewal-theory approach: recursive formulae for the quantities of interest are determined based on the non-resetting version of the process, and solved in the Laplace domain. Since the stochastic process is ergodic, the reward function (2) is determined by the stationary law of the process. This insight proves key in characterising the optimal feedback control, since the system need only be solved in the limit of vanishing Laplace frequency. Our approach is reminiscent of the approach taken in Ref. [37], however, we highlight one subtle yet crucial distinction. In Ref. [37] the law of the process is determined and subsequently the limit of vanishing Laplace frequency is taken. In the general setting of arbitrary $v(x)$, the first step appears all but intractable. Determining the stationary law by solving in the asymptotic limit of vanishing Laplace frequency affords a simplification that, even in the case of arbitrary $v(x)$, remains solvable, as we now demonstrate.

We begin by posing the simpler problem without reset, governed by system (1) after removing the delta function in (1a). Denoting this system by lowercase density $p$ and flux $\phi$, and taking the Laplace transform, the density $\widetilde{p}$ satisfies

$$
s \widetilde{p}+\frac{\partial \widetilde{\phi}}{\partial x}=0,
$$

subject to

$$
\left(\left.\widetilde{\phi}\right|_{x=0}\right)-1=\widetilde{p}(L, s)=0,
$$

for

$$
\widetilde{\phi}=v(x) \widetilde{p}-D \frac{\partial \widetilde{p}}{\partial x} .
$$

We leverage the simpler process $p$ to gain insight into the process of interest $P$. The probability density $H$ of the first-passage time to $x=L$ is given by the rate at which probability is absorbed at the right-hand boundary

$$
H(t)=-\frac{\mathrm{d}}{\mathrm{d} t} \int_{0}^{L} p(x, t) \mathrm{d} x=\left.\phi\right|_{x=L} .
$$

The probability $P$ then satisfies the renewal equation

$$
P(x, t)=p(x, t)+\int_{0}^{t} H(\tau) P(x, t-\tau) \mathrm{d} \tau,
$$

comprising the probability $p$ that the particle has not yet reset and is at position $x$ by time $t$, or has reset at some time $\tau \in(0, t)$ and reached $x$ in the remaining time interval $t-\tau$. Similarly, the average number of resets $\mathcal{N}$ occurring until time $t$ is governed by

$$
\mathcal{N}(t)=\int_{0}^{t} H(\tau)(1+\mathcal{N}(t-\tau)) \mathrm{d} \tau
$$


accounting for the probability of the first reset occurring at an intermediate time $\tau \in(0, t)$, followed by (that is, being one larger than) the average number of resets in the remaining $t-\tau$ after reset.

Both equations (5) and (6) are tractable in the Laplace domain, where we find that

$$
\widetilde{P}(x, s)=\frac{\widetilde{p}(x, s)}{1-\widetilde{H}(s)}, \quad \widetilde{\mathcal{N}}(s)=\frac{\widetilde{H}(s)}{s(1-\widetilde{H}(s))} .
$$

It thus suffices to determine $\widetilde{p}$, since we may determine $\widetilde{H}$ via (4) from which $\widetilde{P}$ and $\widetilde{\mathcal{N}}$ follow from (7). Since the large-time behaviour of the system is determined by the stationary law, it suffices to solve (3) asymptotically in the limit as $s \rightarrow 0$. Posing the expansion

$$
\widetilde{p}(x, s) \sim \widetilde{p}_{0}(x)+s \widetilde{p}_{1}(x)+\cdots,
$$

the leading-order contribution expresses the conservation of probability mass $\widetilde{\phi}_{0}=1$, from which we deduce the leading-order distribution

$$
\widetilde{p}_{0}(x)=\frac{1}{D} \int_{x}^{L} \exp \left(-\frac{1}{D} \int_{x}^{y} v(z) \mathrm{d} z\right) \mathrm{d} y .
$$

At first order we find that $\widetilde{p}_{0}+\mathrm{d} \widetilde{\phi}_{1} / \mathrm{d} x=0$ and therefore

$$
\widetilde{\phi}_{1}=-\int_{0}^{x} \widetilde{p}_{0}(y) \mathrm{d} y .
$$

This equation may be solved for $\widetilde{p}_{1}$, but we will see that knowing only the first-order flux is sufficient.

Equipped with the asymptotic solution up to first order, it follows that

$$
\widetilde{H} \sim\left(\left.\widetilde{\phi}_{0}\right|_{x=L}\right)+s\left(\left.\widetilde{\phi}_{1}\right|_{x=L}\right)=1-s \int_{0}^{L} \widetilde{p}_{0}(x) \mathrm{d} x,
$$

and therefore

$$
\widetilde{P} \sim \frac{1}{s} \frac{\widetilde{p}_{0}}{\int_{0}^{L} \widetilde{p}_{0}(x) \mathrm{d} x}, \quad \quad \widetilde{\mathcal{N}} \sim \frac{1}{s^{2}} \frac{1}{\int_{0}^{L} \widetilde{p}_{0}(x) \mathrm{d} x} .
$$

Inverting the Laplace transforms in (12), the steady distribution and steady reset rate are given by

$$
P(x)=\frac{\widetilde{p}_{0}(x)}{\int_{0}^{L} \widetilde{p}_{0}(x) \mathrm{d} x}, \quad \frac{\mathcal{N}(t)}{t}=\frac{1}{\int_{0}^{L} \widetilde{p}_{0}(x) \mathrm{d} x} .
$$

The long-term reward function $\mathcal{R}$ is thus given by

$$
\mathcal{R}=\frac{1}{L} \int_{0}^{L} x P(x) \mathrm{d} x-\frac{C \mathcal{N}(T)}{T},
$$

where the first term represents the steady average state $x$ and the second term is the steady reset rate penalty. By substituting (13) into (14), and scaling the state $x$ with $L$ and control $v$ with $U$, we obtain a dimensionless form of the reward function

$$
\mathcal{R}=\frac{\int_{0}^{1} X \rho(X ; V) \mathrm{d} X-\hat{C}}{\int_{0}^{1} \rho(X ; V) \mathrm{d} X},
$$




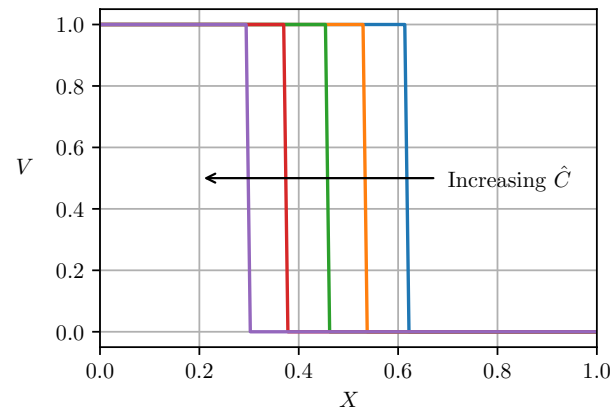

(a) $\hat{C} \in\{0.01,0.03,0.05,0.07,0.09\}$, $\mathrm{Pe}=1, u / U=0$.

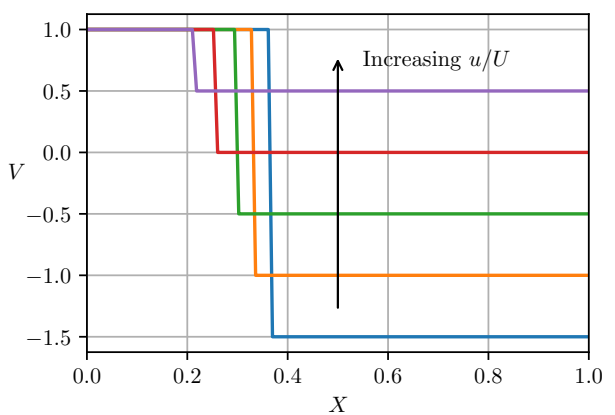

(b) $u / U \in\{-1.5,-1,-0.5,0,0.5\}$, $\mathrm{Pe}=1, \hat{C}=0.1$.

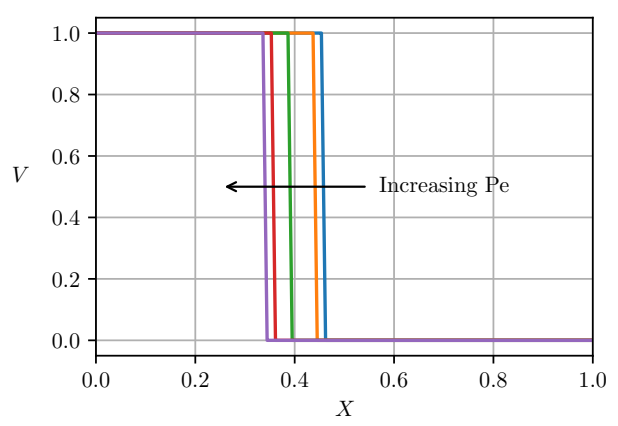

(c) $\mathrm{Pe} \in\{1,3,10,50,100\}, u / U=0, \hat{C}=0.05$.

Figure 1: Optimal scaled feedback controls $V(X)$ solving problem (16) for varying Péclet number Pe, drift ratio $u / U$ and scaled reset cost $\hat{C}$. All optimal controls are bang-bang and non-increasing.

where

$$
\rho(X ; V)=\int_{X}^{1} \exp \left(-\operatorname{Pe} \int_{X}^{Y} V(Z) \mathrm{d} Z\right) \mathrm{d} Y .
$$

for the three free problem parameters: the Péclet number $\mathrm{Pe}=U L / D$ representing the ratio of convection to diffusion, the ratio of the control bounds $u / U$, and the scaled cost $\hat{C}=C D / L^{2}$. The optimal control problem may then be expressed in the form

$$
\max _{V(X) \in[u / U, 1]} \mathcal{R}(V ; \mathrm{Pe}, \hat{C}) .
$$

A first approach is to discretise the domain $X \in[0,1]$ and pose the optimisation problem (16) as a constrained nonlinear program. We approximate the integrals using the trapezoidal rule, encoding the discretisation in the CasADi framework [38], which performs automatic differentiation to provide full gradient information to the IPOPT solver [39]. The numerical results demonstrate that the optimal drift does indeed vary with the state, but in a surprisingly simple way, it adopts the maximum $V(X)=1$ for $X<X_{0}$, and the minimum $V(X)=u / U$ for $X>X_{0}$ throughout the threedimensional parameter space (Fig. 1). In other words, the optimal feedback is a 
bang-bang control (that is, a control that switches between its upper and lower bound, but does not take intermediate values between these bounds [40, §4.4.1]) possessing a single switch.

To shed light on this solution, it is instructive to analyse the first variation of the functional $\mathcal{R}$. For an accessible introduction to the calculus of variations and optimal control, we refer the reader to Ref. [40]. In brief, the first variation is the infinitedimensional analogue of the directional derivative. Just as the directional derivative at a function's optimum must be zero for any direction not exiting the function's domain, so too for the first variation. Our aim is to show that, if the control is not bang-bang, there is a non-exiting "direction" with nonzero first variation, thus, such a control cannot be optimal.

We consider an optimal drift $V$, about which we perturb by $\epsilon W$ for an arbitrary "direction" $W$ in a suitable set of perturbations that we will describe. The first variation, denoted $\delta_{W} \mathcal{R}$, is given from (15) by

$$
\begin{aligned}
\delta_{W} R(V) & :=\left.\frac{\mathrm{d}}{\mathrm{d} \epsilon}[\mathcal{R}(V+\epsilon W)]\right|_{\epsilon=0} \\
& =\frac{\int_{0}^{1}(X-\mathcal{R}(V)) \delta_{W} \rho(X ; V) \mathrm{d} X}{\int_{0}^{1} \rho(X) \mathrm{d} X},
\end{aligned}
$$

where

$$
\delta_{W} \rho(X ; V)=\operatorname{Pe} \int_{X}^{1} \exp \left(\operatorname{Pe} \int_{Y}^{X} V(Z) \mathrm{d} Z\right) \int_{Y}^{X} W(Y) \mathrm{d} Z \mathrm{~d} Y .
$$

Changing the order of integration, we find that $\delta_{W} \mathcal{R}=0$ if and only if

$$
\int_{0}^{1} W(Z) F(Z) \mathrm{d} Z=0,
$$

for

$$
F(Z)=\int_{0}^{Z} \int_{0}^{Y}(X-\mathcal{R}(V)) \exp \left(\operatorname{Pe} \int_{Y}^{X} V(S) \mathrm{d} S\right) \mathrm{d} X \mathrm{~d} Y .
$$

Our aim is to show that $V$ takes only boundary values $V(X) \in\{u / U, 1\}$. By contradiction, assume there is an open interval $\mathcal{I}=\left(X_{1}, X_{4}\right)$ on which $V(X) \in$ $(u / U, 1)$ and $\delta_{W} \mathcal{R}=0$. For the perturbed control to remain admissible, $V+\epsilon W \in$ $[u / U, 1]$, (in other words, for $W$ to be a non-exiting "direction") it suffices to impose that $|W(X)| \leq 1$ and choose $W$ that is nonzero only in an open interval $\mathcal{J}=\left(X_{2}, X_{3}\right)$, where $X_{1}<X_{2}<X_{3}<X_{4}$. It follows from the Fundamental Lemma of the Calculus of Variations that $F(Z) \equiv 0$ on $\mathcal{J}$. Differentiating three times with respect to $Z$ yields $1=0$, which exposes the contradiction.

This argument demonstrates that an optimal $V$ can take only boundary values, and thus the control is bang-bang. Moreover, we argue on physical grounds that $V$ is monotonically non-increasing: if it is optimal to have a drift $V(Y)$ at some state $Y$, it cannot be beneficial to have a lower drift for lower state and so $V(X) \geq V(Y)$ for $X<Y$. We thus deduce that the drift is indeed the bang-bang control with at most a single switch.

Such an optimal feedback lends itself to physical interpretation: the control is acting to steer the system towards an optimal threshold $X_{0}$ that balances between high pay-off and low resets. Holding all other parameters constant, reducing the 
reset penalty increases the threshold $X_{0}$, as lower reset cost allows for elevated risk (Fig. 1a). Similarly, decreasing the lower control bound via $u / U$ increases $X_{0}$, since greater control is afforded to steer clear of resetting (Fig. 1b). Finally, for increasing Pe the optimal threshold $X_{0}$ decreases, as this corresponds to greater capacity to steer toward the gainful region of larger $X$ (Fig. 1c).

To recap, we posed the optimal feedback control problem for a stochastic process under the influence of controllable drift with costly state-dependent reset and linear pay-off. We found that the optimal control is of bang-bang type with a single switch at a threshold state $X_{0}$, taking its upper and lower bounds below and above the threshold, respectively. Therefore, the problem is essentially a one-dimensional optimisation problem of finding the threshold junction $X_{0}$ as a function of the three system parameters.

\section{The two-drift case}

In light of the piecewise-constant control structure, we may substitute into system (1) a drift $v(x)$ of the form

$$
v(x)= \begin{cases}U, & x<x_{0} \\ u, & x>x_{0}\end{cases}
$$

No regularity concerns arise from the discontinuity in $v(x)$. In fact, by integrating over an infinitesimal region containing $x_{0}$, one can show that the system (1) with discontinuous $v(x)(21)$ reduces to solving the convection-diffusion equation

$$
\begin{array}{ll}
\frac{\partial P}{\partial t}+\frac{\partial \Phi_{1}}{\partial x}=\delta(x) \Phi_{2}(L), & 0<x<x_{0}, \\
\frac{\partial P}{\partial t}+\frac{\partial \Phi_{2}}{\partial x}=0, & x_{0}<x<L,
\end{array}
$$

subject to

$$
\begin{aligned}
& \left(\left.\Phi_{1}\right|_{x=0}\right)-\delta(t)=P(L, t)=P(x, 0)=0, \\
& \left(\left.P\right|_{x \uparrow x_{0}}\right)-\left(\left.P\right|_{x \downarrow x_{0}}\right)=\left(\left.\Phi_{1}\right|_{x \uparrow x_{0}}\right)-\left(\left.\Phi_{2}\right|_{x \downarrow x_{0}}\right)=0,
\end{aligned}
$$

where $\Phi_{1}$ and $\Phi_{2}$ denote the fluxes on $x \in\left[0, x_{0}\right)$ and $x \in\left(x_{0}, L\right]$, respectively, given by

$$
\Phi_{1}=U P-D \frac{\partial P}{\partial x}, \quad \Phi_{2}=u P-D \frac{\partial P}{\partial x} .
$$

In regularising the discontinuity two new continuity conditions $(22 d)$ for the density and the flux are introduced at $x=x_{0}$.

Following the same steps as before, but omitting the calculations, we find that the long-term reward $\mathcal{R}$ has an explicit expression as a function of the three system parameters $\mathrm{Pe}, u / U$, and $\hat{C}$. The formula is unwieldy but included in the Appendix for the sake of completeness. The closed-form solution allows us to more readily determine the optimal switching threshold in the three-dimensional parameter space. 


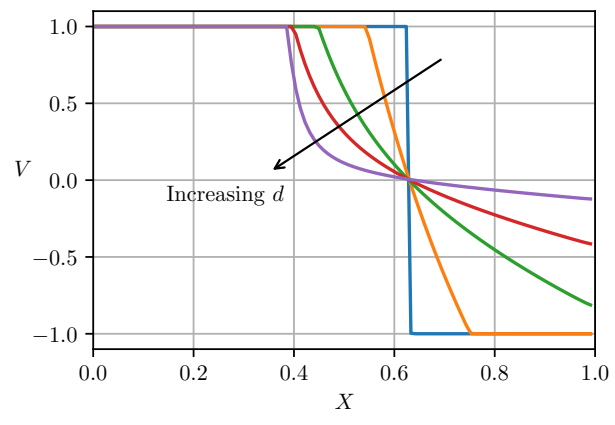

(a) $d \in\{0,0.05,0.15,0.3,1\}$,

$\mathrm{Pe}=1, u / U=-1, \hat{C}=0.01$.

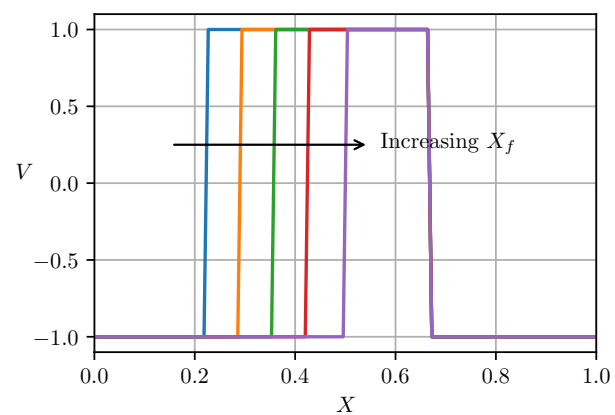

(b) $X_{f} \in\{0.3,0.32,0.34,0.36,0.38\}$, $\mathrm{Pe}=1, u / U=-1, \hat{C}=0.01$.

Figure 2: (a) Optimal control $V(X)$ for control-dependent diffusion $D(V)=1+d V^{2}$. For increasing $d>0$ the control is not bang-bang as it becomes increasingly advantageous to have diminished diffusion $V \approx 0 \in(u / U, 1)$ even at the expense of drift away from the reset point. (b) Non-monotonic bang-bang optimal control for the convex reward function $f(Z)=\left(Z-X_{f}\right)^{2}$.

\section{Beyond linear pay-off and constant diffusion}

In the spirit of making the model more realistic, we seek to relax assumptions made of the underlying stochastic process (1) and reward function (2).

We may consider diffusion that depends on the state $x$ or on the control $v(x)$, denoted $D(x, v(x))$. This can capture the fact that running the system at higher throughput $x$, or accelerating production via $v$, might correlate with varying system fluctuations captured by the diffusion. In this case, the framework described above is unaltered except for the leading-order distribution $\widetilde{p}_{0}(x)$ in $(9)$ is now given by

$$
\widetilde{p}_{0}(x)=\frac{1}{D(x, v(x))} \int_{x}^{L} \exp \left(-\int_{x}^{y} \frac{v(z)}{D(z, v(z))} \mathrm{d} z\right) \mathrm{d} y .
$$

When $D$ is independent of $v$, the variational argument remains entirely intact. However, when $D$ depends on $v$, the argument breaks down and the optimal feedback control may take values within the interval $(u, U)$ (Fig. 2a).

The pay-off need not be linear in $x / L$. It is quite possible that a process exhibits economy (or diseconomy) of scale. We may consider an arbitrary functional $f(x / L)$, since this does not affect the stochastic process, therefore the stationary distribution remains the same, and we need only update the integrand in (14) from $(x / L) P(x)$ to $f(x / L) P(x)$. Higher order moments of $x$, that is, $f(Z)=Z^{n}$ for integer $n \geq 2$, admit closed forms. Moreover, the variational argument is preserved for any strictly monotonic or strictly concave $f$. For the broader class of functions $f$ that are not stationary on any open interval an optimal control is still bang-bang, however, it need not be monotonic in $x$ (Fig. $2 \mathrm{~b}$ ), and thus does not necessarily reduce to the two-drift case. 


\section{Conclusion}

Leveraging the process ergodicity and long-term nature of the objective function, we demonstrated how the optimal feedback control of a one-dimensional diffusion process with resetting may be reduced to a tractable maximisation problem, thereby circumventing the need to solve the Hamilton-Jacobi-Bellman equation (a nonlinear parabolic partial differential equation governing the optimal reward from any system state, from which the optimal control may be derived [41], but for which finding a solution proves "a very difficult task" [40, §5.2]). The bang-bang form of the control is reminiscent of (non-singular) deterministic optimal control problems where the Hamiltonian is affine with respect to the control, just as here the stochastic dynamics governing the process evolution is affine in the control. The simplest problem formulation admits a solution that is concretely implementable in practice. Generalising the process and objective function reveals a rich optimisation landscape that gives rise to a more intricate tapestry of optimal controls.

Looking forward, it would be intriguing to extend the optimal control problem to the settings proposed in Ref. [37], such as delays upon reset and multiple coupled stochastic processes. Furthermore, quantifying the influence of transient effects, such as a finite time horizon and a time-dependent pay-off, would also be important steps towards making the model more widely applicable in real-world settings.

\section{Appendix}

The long-term reward given in (2), corresponding to the discontinuous control (21), or equivalently, the two-drift system (22), is given by

$$
\mathcal{R}=\frac{A_{n}}{A_{d}}-\hat{C} \frac{N_{n}}{N_{d}},
$$

where

$$
\begin{aligned}
A_{n}= & \mathrm{Pe}_{u}^{2}\left(\mathrm{Pe}-\mathrm{Pe}_{u}\right) \mathrm{e}^{-\mathrm{Pe}_{0}}-\mathrm{PePe}_{u}^{2} \mathrm{e}^{\mathrm{Pe}_{u} X_{0}-\mathrm{Pe} X_{0}-\mathrm{Pe}_{u}} \\
& +\mathrm{Pe}\left(\mathrm{Pe}-\mathrm{Pe}_{u}\right)\left(X_{0} \mathrm{PePe}_{u}-\mathrm{Pe}-\mathrm{Pe}_{u}\right) \mathrm{e}^{-\mathrm{Pe}_{u}\left(1-X_{0}\right)} \\
& +\mathrm{Pe}^{3}+\mathrm{Pe}_{u}^{3}+\frac{1}{2} \mathrm{Pe}^{3} \mathrm{Pe}_{u}^{2}-\mathrm{PePe}_{u}^{2}-\mathrm{Pe}^{3} \mathrm{Pe}_{u} \\
& -\frac{1}{2} X_{0} \mathrm{PePe}_{u}^{2}\left(\mathrm{Pe}-\mathrm{Pe}_{u}\right)\left(X_{0} \mathrm{Pe}-2\right), \\
A_{d}= & \mathrm{PePe}_{u}\left(\mathrm{Pe}\left(\mathrm{Pe}-\mathrm{Pe}_{u}\right) \mathrm{e}^{-\mathrm{Pe}_{u}\left(1-X_{0}\right)}-\mathrm{Pe}_{u}\left(\mathrm{Pe}-\mathrm{Pe}_{u}\right) \mathrm{e}^{-\mathrm{Pe}_{0}}\right. \\
& +\mathrm{PePe}_{u} \mathrm{e}^{\mathrm{Pe}_{u} X_{0}-\mathrm{Pe}_{0}-\mathrm{Pe}_{u}}+\mathrm{PePe}_{u}+\mathrm{Pe}^{2} \mathrm{Pe}_{u}-\mathrm{Pe}^{2}-\mathrm{Pe}_{u}^{2} \\
& \left.-\mathrm{PePe}_{u} X_{0}\left(\mathrm{Pe}-\mathrm{Pe}_{u}\right)\right), \\
N_{n}= & \mathrm{Pe}^{2} \mathrm{Pe}_{u}^{2}, \\
N_{d}= & \mathrm{PePe}_{u}\left(1+\mathrm{Pe}-X_{0}\left(\mathrm{Pe}-\mathrm{Pe}_{u}\right)\right)-\mathrm{Pe}^{2}-\mathrm{Pe}_{u}^{2} \\
& -\mathrm{Pe}_{u}\left(\mathrm{Pe}-\mathrm{Pe}_{u}\right) \mathrm{e}^{-\mathrm{Pe}_{0}}+\mathrm{Pe}\left(\mathrm{Pe}-\mathrm{Pe}_{u}\right) \mathrm{e}^{-\mathrm{Pe}_{u}\left(1-X_{0}\right)} \\
& +\mathrm{PePe}_{u} \mathrm{e}^{-\mathrm{Pe}_{u}-\mathrm{Pe}_{0}+\mathrm{Pe}_{u} X_{0}},
\end{aligned}
$$

for $\mathrm{Pe}_{u}=(u / U) \mathrm{Pe}$. 
Optimal feedback control in first-passage resetting

\section{References}

[1] C. Gardiner. Stochastic Methods: A Handbook for the Natural and Social Sciences. Springer Berlin Heidelberg, third edition, 2009.

[2] W. Weidlich and G. Haag. Concepts and models of a quantitative sociology: the dynamics of interacting populations, volume 14. Springer Science \& Business Media, 2012.

[3] O. Bénichou, M. Moreau, P.-H. Suet, and R. Voituriez. Intermittent search process and teleportation. J. Chem. Phys., 126(23):234109, 2007.

[4] É. Roldán, A. Lisica, D. Sánchez-Taltavull, and S. W. Grill. Stochastic resetting in backtrack recovery by rna polymerases. Phys. Rev. E, 93:062411, Jun 2016.

[5] I. Eliazar, T. Koren, and J. Klafter. Searching circular DNA strands. J. Phys.-Condens. Mat., 19(6):065140, Jan 2007.

[6] P. J. Brockwell, J. Gani, and S. I. Resnick. Birth, immigration and catastrophe processes. Adv. Appl. Probab., 14(4):709-731, 1982.

[7] R. Bartoszynski, W. J. Buehler, W. Chan, and D. K. Pearl. Population processes under the influence of disasters occurring independently of population size. J. Math. Biol., 27(2):167$178,1989$.

[8] E. G. Kyriakidis. Stationary probabilities for a simple immigration-birth-death process under the influence of total catastrophes. Stat. Probabil. Lett., 20(3):239-240, 1994.

[9] P. J. Brockwell, J. Gani, and S. I. Resnick. Birth, immigration and catastrophe processes. Adv. Appl. Probab., 14(4):709-731, 1982.

[10] B. Krishna Kumar and D. Arivudainambi. Transient solution of an $M / M / 1$ queue with catastrophes. Comput. Math. Appl., 40(10):1233-1240, 2000.

[11] B. Krishna Kumar and S. Pavai Madheswari. Transient behaviour of the $M / M / 2$ queue with catastrophes. Statistica, 62(1):129-136, 2002.

[12] X. Chao and Y. Zheng. Transient analysis of immigration birth-death processes with total catastrophes. Probab. Eng. Inform. Sc., 17(1):83-106, 2003.

[13] M. Luby, A. Sinclair, and D. Zuckerman. Optimal speedup of las vegas algorithms. Inform. Process. Lett., 47(4):173-180, 1993.

[14] C. P. Gomes, B. Selman, N. Crato, and H. Kautz. Heavy-tailed phenomena in satisfiability and constraint satisfaction problems. J. Autom. Reasoning, 24(1-2):67-100, 2000.

[15] C. P. Gomes and B. Selman. Algorithm portfolios. Artif. Intell., 126(1):43-62, 2001. Tradeoffs under Bounded Resources.

[16] L. Kusmierz, S. N. Majumdar, S. Sabhapandit, and G. Schehr. First order transition for the optimal search time of lévy flights with resetting. Phys. Rev. Lett., 113:220602, Nov 2014.

[17] M. R. Evans and S. N. Majumdar. Diffusion with stochastic resetting. Phys. Rev. Lett., 106:160601, Apr 2011.

[18] M. R. Evans and S. N. Majumdar. Diffusion with optimal resetting. J. Phys. A-Math. Theor., 44(43):435001, 2011.

[19] E. Gelenbe. Search in unknown random environments. Phys. Rev. E, 82:061112, Dec 2010

[20] M. R. Evans and S. N. Majumdar. Diffusion with resetting in arbitrary spatial dimension. $J$. Phys. A-Math. Theor., 47(28):285001, Jun 2014.

[21] A. Falcón-Cortés, D. Boyer, L. Giuggioli, and S. N. Majumdar. Localization transition induced by learning in random searches. Phys. Rev. Lett., 119:140603, Oct 2017.

[22] A. Pal, I. Eliazar, and S. Reuveni. First passage under restart with branching. Phys. Rev. Lett., 122:020602, Jan 2019.

[23] S. Reuveni. Optimal stochastic restart renders fluctuations in first passage times universal. Phys. Rev. Lett., 116:170601, Apr 2016.

[24] A. Pal and S. Reuveni. First passage under restart. Phys. Rev. Lett., 118:030603, Jan 2017.

[25] S. C. Manrubia and D. H. Zanette. Stochastic multiplicative processes with reset events. Phys. Rev. E, 59:4945-4948, May 1999.

[26] M. Montero, A. Masó-Puigdellosas, and J. Villarroel. Continuous-time random walks with reset events. Eur. Phys. J. B, 90(9):176, 2017.

[27] M. R. Evans and S. N. Majumdar. Run and tumble particle under resetting: a renewal approach. J. Phys. A-Math. Theor., 51(47):475003, 2018.

[28] M. R. Evans, S. N. Majumdar, and G. Schehr. Stochastic resetting and applications. J. Phys. A-Math. Theor., 53(19):193001, Apr 2020.

[29] A. Pal, A. Kundu, and M. R. Evans. Diffusion under time-dependent resetting. J. Phys. A-Math. Theor., 49(22):225001, Apr 2016.

[30] U. Bhat, C. De Bacco, and S. Redner. Stochastic search with poisson and deterministic resetting. J. Stat. Mech.-Theory E., 2016(8):083401, Aug 2016. 
[31] A. Chechkin and I. M. Sokolov. Random search with resetting: A unified renewal approach. Phys. Rev. Lett., 121:050601, Aug 2018.

[32] E. G. Kyriakidis and A. Abakuks. Optimal pest control through catastrophes. J. Appl. Probab., 26(4):873-879, 1989.

[33] Y. J. Leung, W. V. Li, and Rakesh. Spectral analysis of brownian motion with jump boundary. P. Am. Math. Soc., 136(12):4427-4436, 2008.

[34] I. Ben-Ari and R. G. Pinsky. Ergodic behavior of diffusions with random jumps from the boundary. Stoch. Proc. Appl., 119(3):864-881, 2009.

[35] I. Grigorescu and M. Kang. Brownian motion on the figure eight. J. Theor. Probab., 15(3):817$844,2002$.

[36] I. Grigorescu and M. Kang. Ergodic properties of multidimensional brownian motion with rebirth. Electronic Journal of Probability, 12:1299-1322, 2007.

[37] B. De Bruyne, J. Randon-Furling, and S. Redner. Optimization in first-passage resetting. Phys. Rev. Lett., 125:050602, Jul 2020.

[38] J. A. E. Andersson, J. Gillis, G. Horn, J. B. Rawlings, and M. Diehl. CasADi: a software framework for nonlinear optimization and optimal control. Math. Program. Comput., 11(1):136, 2019.

[39] A. Wächter and L. T. Biegler. On the implementation of an interior-point filter line-search algorithm for large-scale nonlinear programming. Math. Program., 106(1):25-57, March 2006.

[40] D. Liberzon. Calculus of variations and optimal control theory: A concise introduction. Princeton University Press, 2011.

[41] B. Oksendal. Stochastic differential equations: an introduction with applications. Springer Science \& Business Media, 2013. 